\title{
Monseigneur Ignace Bourget et Monseigneur Jean Langevin face à la Confédération
}

\section{Léon Pouliot}

Volume 34, 1967

URI : https://id.erudit.org/iderudit/1007419ar

DOI : https://doi.org/10.7202/1007419ar

Aller au sommaire du numéro

Éditeur(s)

Les Éditions Historia Ecclesiæ Catholicæ Canadensis Inc.

ISSN

0318-6172 (imprimé)

1927-7067 (numérique)

Découvrir la revue

Citer cet article

Pouliot, L. (1967). Monseigneur Ignace Bourget et Monseigneur Jean Langevin face à la Confédération. Sessions d'étude - Société canadienne d'histoire de

l'Église catholique, 34, 33-40. https://doi.org/10.7202/1007419ar

Tous droits réservés @ Les Éditions Historia Ecclesiæ Catholicæ Canadensis Inc., 1968
Ce document est protégé par la loi sur le droit d'auteur. L'utilisation des services d'Érudit (y compris la reproduction) est assujettie à sa politique d'utilisation que vous pouvez consulter en ligne.

https://apropos.erudit.org/fr/usagers/politique-dutilisation/ 


\section{Monseigneur Ignace Bourget et Monseigneur Jean Langevin face à la Confédération}

On m'a demandé de vous parler de Mgr Ignace Bourget, évêque de Montréal ${ }^{1}$, et de Mgr Jean Langevin, premier évêque de Rimouski, face à la Confédération.

\section{Mgr Bourget.}

Signalons d'abord les faits et les documents et plaçons-les dans leur ordre chronologique : I. Avant la proclamation royale du 22 mai 1867 , qui fait de la Confédération la loi du pays; II. Après la proclamation royale.

\section{I}

Avant la proclamation royale.

Du printemps de 1864, date où la Confédération est mise à l'ordre du jour et acceptée par les deux partis politiques, au printemps de 1867, date où elle devient la loi du pays, il n'existe aucune déclaration publique de Mgr Bourget.

Le 14 novembre 1864, il part pour Rome. La Confédération est alors le grand sujet d'actualité. Les conférences de Charlottetown et de Québec ont eu lieu et à la session de 1864-1865, le parlement sera invité à ratifier ou à rejeter le projet.

Pour préparer les esprits, dans le Globe de Toronto, George Brown chante sur tous les tons l'éloge de la Confédération. A Montréal, La Minerve, organe des conservateurs, qui paraissait trois fois la semaine, devient quotidienne le 19 septembre 1864, et elle est, elle aussi, fédéraliste. Mgr Bourget suit de près les événements. Dans la circulaire où il annonce son voyage en Europe, il écrit :

Nous prierons d'une manière particulière pour le pays, dans ce temps de mouvement et de réforme constitutionnelle, afin que la divine Providence, qui a toujours veillé sur ses intérêts avec une bonté maternelle, dirige toutes les opérations de nos hommes publics et qu'il ne nous arrive rien qui puisse nuire à la Religion et au bienêtre de notre chère patrie 2 .

Nous avons d'ailleurs le témoignage de son grand vicaire, M. Truteau, affirmant qu'avant son départ pour Rome, l'évêque n'avait pas encore

1 Nous avons déjà étudié ce sujet à la réunion de Toronto, en 1959. Rapp. Soc. Can. Hist. de l'Eglise cath., 1959, 31-41.

2 Mandements des Evêques de Montréal (MEM), 5, 33. 
pris parti ${ }^{3}$. Mais s'il n'existe pas de document public de Mgr Bourget sur le sujet, sa correspondance nous invite à penser qu'il n'était pas sans inquiétude. Ce qu'il redoutait, ce n'était pas la forme de gouvernement, mais les circonstances dans lesquelles elle voyait le jour. Comme tant d'autres, il était rêveur devant la coalition inattendue de Cartier avec George Brown, considéré à tort ou à raison comme l'ennemi irréductible des Canadiens français et des institutions catholiques. Telle fut également la réaction de George Clerk, rédacteur du True Witness, hebdomadaire catholique de langue anglaise. Le premier article de Clerk, anrès la coalition Cartier-Brown, śtonna M. Cazcau, vicairce-gćnćrāl de Québec, qui le signala à l'attention de Mgr Bourget. La réponse de celui-ci mérite d'être lue, parce qu'elle nous livre sa pensée à la date du 28 juin 1864. Quand il rédige cette lettre, l'évêque est à Vaudreuil, en tournée pastorale et donc surchargé de travail ${ }^{4}$. Une de ses phrases, claire pour lui peut paraître obscure au lecteur d'aujourd'hui. Quand il écrit : "Pour ma part, j'aimerais mieux que M. Clerk ne fût plus qu'un chien mort au lieu d'être un chien muet », que veut-il dire ? Voici le sens que nous y lisons, en interprétant cette phrase par le contexte général. L'évêque a dit plus haut que $M$. Clerk était un chien de garde qui doit aboyer devant le danger. Si M. Clerk n'aboie pas parce qu'il est mort, on ne peut le lui reprocher. S'il est bel et bien vivant et qu'il n'aboie pas, il ne remplit pas son rôle. Ce qui revient à dire que, dans la pensée de Mgr Bourget, Clerk n'a pas outrepassé la liberté permise à un journaliste catholique militant en attirant l'attention sur les dangers de la coalition Cartier-Brown; il ne mérite pas le blâme que semblait lui adresser M. Cazeau.

Au début de 1867, Cartier envoie de Londres à Mgr Bourget une copie de l'Acte de la Confédération. L'évêque s'empresse d'accuser réception; mais il ne se prononce pas sur le fond du problème; il s'abstient même d'écrire le mot de Confédération, et cela pour la raison très simple que celle-ci n'étant pas encore la loi du pays, reste sujette à la libre discussion des citoyens ${ }^{5}$.

Après la proclamation royale.

Par une proclamation royale du 22 mai 1867, la Confédération devenait la loi du pays. Trois jours plus tard, Mgr Bourget adressait une circulaire à son clergé "sur la conduite à tenir pendant les prochaines élections ${ }^{6}$ ", les premières de la Confédération. Et cette circulaire est le premier document inspiré à un évêque du Bas-Canada par la nouvelle forme de gouvernement. A la vérité, la Confédération

3 Archives de la Chancellerie, Archevêché de Montréal, Registres des Lettres de Mgr Bourget (RLB), 14, 288-290. M. Truteau à M. Cazeau, 20 février 1865.

4 RLB, 13, 573-574. Voir texte à l'Appendice I.

5 RLB, 16, 103. Mgr Bourget à Cartier, 11 mars 1867.

6 MEM, 5, 212. 
n'est pas ici nommée. Mais il est impossible de nous méprendre sur la pensée de l'évêque de Montréal. Il demande deux choses à ses prêtres : 1. mettre les fidèles en garde contre tous les dangers moraux que comportent les élections; 2 . éclairer les consciences par le rappel des bons principes. Et il écrit :

Un de ces vrais principes pour tous les catholiques sincères est que tous les sujets sont obligés en conscience de se soumettre à tout gouvernement légitimement établi; et que ce serait un excès condamnable de travailler à le renverser par la violence ou autres mauvais moyens.

Ce texte vise manifestement ceux-là et ceux-là seuls qui ne se soumettent pas à la Confédération, puisque celle-ci est désormais « le gouvernement légitimement établi ».

A la fin de juin 1867, les évêques de Québec, Saint-Hyacinthe, Trois-Rivières et Rimouski ont publié soit une Pastorale soit un mandement sur la Confédération. Seul, Mgr Bourget ne s'est pas encore adressé à ses fidèles. Pourquoi ? Sa position est plus délicate que celle de ses confrères. Montréal avait été le grand centre de résistance au nouveau mode de gouvernement. Et parmi les adversaires du projet, il y avait d'excellents catholiques, tels George Clerk, rédacteur du True Witness et le juriste universellement estimé qu'était Côme-Séraphin Cherrier. Après la proclamation royale, la Confédération était devenue le gouvernement légitime, celui que "tout catholique sincère " doit accepter. Il convenait de le rappeler assurément, et l'évêque de Montréal avait confié cette tâche au clergé paroissial. Qu'importe au " catholique sincère » que la doctrine de l'Eglise lui soit connue par la voix du curé ou celle de l'évêque?

Mais la campagne électorale bat son plein. Et parmi les candidats en lice, il y en a qui se promettent de renverser la Confédération quand ils seront élus, et qui exploitent en leur faveur le silence de Mgr Bourget. Tous les autres évêques ont parlé, et lui il se tait. Pourquoi sinon parce qu'il est hostile? De cette prétendue division de la hiérarchie, on concluait que la doctrine de la soumission au pouvoir établi n'était pas si sûre que cela et que les catholiques pouvaient en toute tranquillité de conscience voter pour les candidats anti-fédéralistes.

A ce point précis du débat, Mgr Bourget intervient. Car on lui prête des sentiments qu'il n'a pas. Sa Pastorale est du 25 juillet. En son fond essentiel, elle ne diffère pas des documents publiés par les autres évêques. Elle est plus strictement doctrinale en ce sens qu'elle ne porte aucun jugement sur la Confédération comme telle; celle-ci n'est pas comparée non plus à l'Union qu'elle remplace ni à l'annexion aux Etats-Unis que d'autres voudraient lui substituer. Il ne parle pas, non plus, du salut de la nation canadienne-française. Il rappelle que nos ancêtres se sont soumis à toutes les formes de gouvernement qui ont régi le pays sans cesser d'être eux-mêmes. L'Eglise a fait de même et aujourd'hui, elle accepte la Confédération sans réplique; et cela pour la simple raison qu'elle est désormais le gouvernement légitimement établi. 
Cette Pastorale ne parut pas assez claire à Mgr Larocque, évêque de Saint-Hyacinthe. Il demande à l'évêque de Montréal une mise au point plus explicite contre les "libéraux exagérés". Il ne fut pas exaucé. Mgr Bourget avait soumis le contenu de sa Pastorale à l'archevêque. Il avait dit ce qu'il fallait dire. En se limitant au seul rappel des principes, il a élevé le problème au-dessus des passions politiques. Il a fait son devoir, tout son devoir. Aux électeurs catholiques d'en faire autant. Car ils savent où est leur devoir, puisque, aujourd'hui, l'Eglise accepte sans réplique la Confédération.

\section{Monseigneur Jean Langevin.}

Nommé évêque de Rimouski le 15 janvier 1867, il était sacré le $I^{\text {er }}$ mai suivant. Il était le frère d'un des Pères de la Confédération. Son mandement est du 13 juin. On y lit ce qui suit :

La Constitution qui fonde ainsi au Nord des Etats-Unis un grand et riche empire, a été, Nous le croyons sincèrement, amenée providentiellement par une suite de circonstances exceptionnelles. Les rouages de la machine gouvernementale ne pouvaient plus fonctionner; mille rivalités de races, d'intérêts politiques ou sectionnels nous menaçaient d'une anarchie complète, lorsque plusieurs de nos hommes d'Etat les plus éminents ont formé le projet, pour mettre fin à ces difficultés interminables et toujours renaissantes, d'agrandir leur sphère d'action et d'unir en un puissant Etat des provinces qui, dans leur isolement, n'avaient que bien peu de moyens de développer leurs ressources. C'est ce projet, fruit de mûres délibérations, qui a été soumis à l'approbation des Parlements provinciaux et à celle du Parlement impérial et qui est devenue dans toutes ses dispositions essentielles la loi du pays?.

Il rappelle aux fidèles l'obligation de conscience qu'ils ont de voter pour le candidat qui leur paraît le plus apte à promouvoir le bien public, car de ce suffrage dépend beaucoup "le salut de notre pays aussi bien que la conservation de tout ce qui nous est cher comme nation, notre Religion, notre Langue et nos Institutions ».

Il met les fidèles en garde contre les annexionnistes : "S'ils réussissaient dans leurs sinistres projets, ce qu'à Dieu ne plaise. ce serait, à moins d'un miracle de la Providence, la ruine de notre peuple, la perte de nos mœurs, de nos coutumes, l'anéantissement de notre nationalité. " En conséquence, il faut exiger des candidats une promesse formelle et positive de soutenir la nouvelle constitution.

Le Mandement suppose évidemment connue la doctrine de l'Eglise sur la soumission aux gouvernements légitimes, mais il n'en fait pas explicitement le motif d'adhésion à la Confédération. Ce motif se trouve dans la plus grande chance de survie nationale et religieuse qu'offre le nouveau mode de gouvernement.

$$
\text { Léon Pouliot. s.j. }
$$

7 Mandements des Evêques de Rimouski, Langevin, Jean (Mgr), Rimouski, 1872, Vol. 1, p. 17-22. Voir texte à l'Appendice II. 


\section{A P P E N D I C E I}

\section{Monseigneur Bourget à M. C.-F. Cazeau}

Vaudreuil, 28 juin 1864.

J'ai lu l'article du True Witness concernant le ministère qui est à se former; et je n'y vois qu'un avis important donné à ceux qui croient pouvoir contracter l'alliance en question. $\mathbf{M}^{r}$ Clerk est le chien qui doit aboyer quand il sent quelque ennemi du catholicisme approcher. Or, il se trouve que $\mathrm{M}^{r}$ Brown a toujours travaillé à couvrir de boue le catholicisme dont il s'est toujours montré un ennemi furibond. Si cela n'est pas vrai, il (M. Clerk) a eu grand tort de jeter le cri d'alarme. Mais si cela est vrai, on ne peut lui faire un crime d'avertir que ceux qui aujourd'hui donnent la main à ce mortel ennemi de la Religion ont à y prendre garde. Si dans la suite on a à déplorer cette alliance, les catholiques n'auront toujours pas à se reprocher d'avoir été endormis en présence d'un si grand danger ou dupés par un homme qui jusqu'ici ne s'est distingué que par sa haine contre le Bas-Canada ct ses institutions religieuses. Pour ma part, j'aimerais mieux que $\mathbf{M}^{\mathbf{r}}$ Clerk ne fût plus qu'un chien mort au lieu d'être un chien muet.

Maintenant, je vous avouerai franchement, $\mathbf{M}^{\mathrm{r}}$ le Grand Vicaire, que le projet d'alliance entre le ministère actuel et $M^{r}$ Brown m'a jeté dans une profonde tristesse, parce que $\mathrm{j}$ 'y vois l'humiliation du catholicisme; et dans mon opinion, s'il n'y a pas pour nous d'autre planche de salut que cette sinistre alliance, il faut tout de bon se préparer au naufrage.

Mais j'élève plus haut mes espérances, et je crois que la divine Providence qui a toujours si visiblement protégé notre pays viendra encore à son secours.

Je vous dirai en terminant que je garde le silence pour le moment sur la formation du ministère. en attendant que ses actes nous mettent à même de le juger par des faits palpables et évidents. Je me propose d'obéir à son gouvernement, quoiqu'il ne m'inspire aucune confiance. $\mathrm{Si}$, plus tard, les intérêts du catholicisme sont en danger. je ferai toute l'opposition que peut faire un évêque à un gouvernement qui oublie ses devoirs.

RLB.13, 573-574. 


\title{
A P P E N D I C E I I
}

\author{
MA N D E M E N T
}

de

L'ÉVÊQUE DE SAINT-GERMAIN DE RIMOUSKI,

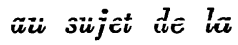

PROCLAMATION DE LA CONFÉDÉRATION.

J E A N L A N G E V I N,

par la miséricorde de Dieu et la Grâce du Saint-Siège

Apostolique, Evêque de Saint-Germain de Rimouski,

\section{Au clergé et aux fidèles du Diocèse}

\section{SALUT ET BÉNÉDICTION EN NOTRE-SEIGNEUR.}

Un événement de la plus haute gravité et susceptible des conséquences les plus importantes pour ce pays va s'accomplir dans quelques jours, Nos Chers Frères. En vertu d'une proclamation de Notre TrèsGracieuse Souveraine, un Acte récent du Parlement Impérial unissant en une seule Puissance les provinces du Canada, de la Nouvelle-Ecosse et du Nouveau-Brunswick, prendra effet le $1^{\text {er }}$ jour de juillet prochain.

Vous le comprenez, Nos bien-aimés Frères, c'est là toute une révolution accomplie paisiblement et d'une manière réfléchie; c'est là un changement de Constitution qui fait entrer ces colonies de la GrandeBretagne dans une voie nouvelle, qui ouvre devant elles un avenir bien vaste, qui étend prodigieusement leur horizon jusqu'alors si borné.

Dans un moment si solennel pour la patrie commune, vous tournez naturellement les yeux vers vos premiers Pasteurs, Fidèles de l'Eglise du Canada, pour en recevoir une direction sage et salutaire. Jamais, par le passé, la voix de vos Evêques n'a manqué de se faire entendre dans toutes les conjonctures importantes : jamais ils n'ont hésité à vous indiquer la ligne de conduite à suivre, à se mettre à votre tête, à prendre la défense de vos intérêts; jamais vous ne vous êtes repentis de vous être montrés dociles à leurs conseils.

Aujourd'hui donc, Nos Chers Frères, vous seriez étonnés, alarmés, si vos guides naturels ne se prononçaient sur les événements qui s'accomplissent à notre égard. Aussi croyons-Nous remplir un des devoirs de notre charge sacree, en venant vous offrir quelques avis, que vous accueillerez, comme toujours, avec empressement et respect.

La Constitution qui fonde ainsi au Nord des Etats-Unis un grand et riche empire, a été, Nous le croyons sincèrement, amenée providentiellement par une suite de circonstances tout à fait exceptionnelles. Les rouages de la machine gouvernementale ne pouvaient plus fonc- 
tionner; mille rivalités de races, de croyances religieuses, d'intérêts politiques ou sectionnels, nous menaçaient d'une anarchie complète; lorsque plusieurs de nos hommes d'Etat les plus éminents ont formé le projet, pour mettre fin à ces difficultés interminables et toujours renaissantes, d'agrandir leur sphère d'action, et d'unir en un puissant Etat des Provinces qui, dans leur isolement, n'avaient que bien peu de moyens de développer leurs ressources. C'est ce projet, fruit de mûres délibérations, qui a été soumis à l'approbation des Parlements Provinciaux et à celle du Parlement Impérial, et qui est devenu, dans toutes ses dispositions essentielles, la loi du pays.

Vous la respecterez donc, Nos Chers Frères, cette nouvelle Constitution qui vous est donnée, comme l'expression de la volonté suprême du Législateur, de l'Autorité légitime, et par conséquent de celle de Dieu même. Ne vous dit-il pas en effet au Livre des Proverbes : "C'est par moi que règnent les Rois, et que les Législateurs font des lois justes : c'est par moi que les Princes commandent, et que ceux qui ont le pouvoir rendent la justice." Per me Reges regnant et legum conditores justa decernunt: per me Principes imperant et potentes decernunt justitiam. - Prov. VIII, 15, 16.

Dans les élections prochaines, vous considérerez comme une obligation de conscience de choisir avec soin ceux qui doivent vous représenter, soit dans la Chambre des Communes, soit dans le Parlement local. De ce choix fait avec discernement, sans passions mesquines, sans préférence purement personnelle, avec l'unique désir du bien public, avec un véritable patriotisme en un mot, dépend beaucoup le salut de notre pays, aussi bien que la conservation de tout ce qui nous est cher comme nation, notre Religion, notre Langue, nos Institutions.

Nous vous le déclarons hautement, Nos Chers Frères : le misérable qui, dans une affaire de cette gravité, se laisserait tenter par un vil intérêt, qui vendrait son vote au plus offrant, qui se déclarerait en faveur du Candidat assez misérable pour le gagner avec de la boisson forte : celui-là serait l'homme le plus bas et le plus dégradé, indigne de jouir des privilèges d'un électeur; ce serait un monstre dont la Patrie et l'Eglise auraient à rougir.

Vous allez donc choisir, Nos Chers Frères, des Représentants capables de soutenir vos intérêts et de vous faire honneur par leurs principes honnêtes, par leur éducation, par leur expérience des affaires publiques. Ils devront vous promettre de travailler franchement et cordialement à faire fonctionner le nouvel ordre de choses, et à seconder à cet effet ceux qui vont être appelés à l'inaugurer. Vous vous défierez, s'il s'en rencontrait parmi vous, de ces esprits mécontents qui rêvent pour le Canada le bonheur et la prospérité dans l'annexion à un pays voisin. S'ils réussissaient dans leurs sinistres projets, ce qu'à Dieu ne plaise. ce serait, à moins d'un miracle de la Providence, la ruine de notre peuple, la perte de nos mœurs, de nos coutumes, de notre langue, l'anéantissement de notre nationalité. Vous exigerez donc des Candidats une déclaration explicite et formelle de principes, l'engagement positif de soutenir la nouvelle Constitution. 
Surtout, Nos Chers Frères, au nom de vos intérêts les plus précieux, restez unis, serrez vos rangs, marchez ensemble sous la direction éclairée et paternelle de ceux qui doivent vous conduire. Nous diviser dans ce moment serait nous suicider, nous faire une blessure mortelle. Si nous voulons avoir, dans les conseils de la nouvelle Nation Canadienne, dans le Parlement de la Confédération, notre juste et légitime influence; dans la balance du gouvernement le poids auquel nous donne droit le chiffre de notre population; demeurons unis, encore une fois, d'esprit, de cœur, de volonté.

Une autre chose que Nous vous recommandons instamment. Nos bien chers Frères, c'est le respect pour la sainteté du serment. Trop souvent, quand on se laisse emporter à l'esprit de parti, on oublie combien le Nom du Seigneur est sacré et terrible : sanctum et terribile Nomen ejus (Ps. 110), et comme il ne faut le prendre à témoin que pour des choses vraies et justes.

Mais comme Dieu est le Maître des nations aussi bien que des individus, que c'est Lui qui les élève et les abaisse à son gré, qui les fonde et les dissipe, Nous jugeons qu'il est juste et raisonnable. dans un moment si solennel. d'élever nos mains et nos cœurs vers le trône de sa bonté, et d'implorer tous ensemble sa bénédiction pour notre Patrie.

A ces causes, Nous avons réglé et ordonné, réglons et ordonnons ce qui suit :

$1^{\circ}$ Lundi, le $1^{\text {er }}$ juillet prochain, il sera chanté à cette intention dans toutes les paroisses et missions de ce Diocèse une grand'messe solennelle de l'octave de Saint-Jean-Baptiste, que l'on fera suivre de l'hymne Veni Creator, du verset Emitte, et de l'Oraison Deus, qui cordı fidelium.

$2^{\circ}$ Le présent Mandement y sera lu au prône le premier dimanche ou jour de fête après sa réception.

Donné à Saint-Germain de Rimouski, en notre demeure épiscopale, sous notre seing, le sceau du Diocèse, et le contre seing de notre secrétaire pro tempore, le treizième jour de juin 1867.

\author{
JEAN, \\ Evếque de Saint-Germain de Rimouski, \\ Par Monseigneur,
}

CHARLES ROULEAU, Eccl. Secrétaire pro tempore. 